\title{
From Self-Assembled Vesicles to Protocells
}

\author{
Irene A. Chen ${ }^{1}$ and Peter Walde ${ }^{2}$ \\ ${ }^{1}$ FAS Center for Systems Biology, Harvard University, Cambridge, Massachusetts 02138 \\ ${ }^{2}$ Department of Materials, ETH Zurich, CH-8093, Zurich, Switzerland \\ Correspondence: ichen@Isdiv.harvard.edu
}

Self-assembled vesicles are essential components of primitive cells. We review the importance of vesicles during the origins of life, fundamental thermodynamics and kinetics of self-assembly, and experimental models of simple vesicles, focusing on prebiotically plausible fatty acids and their derivatives. We review recent work on interactions of simple vesicles with RNA and other studies of the transition from vesicles to protocells. Finally we discuss current challenges in understanding the biophysics of protocells, as well as conceptual questions in information transmission and self-replication.

For synthetic biologists, a useful operational F definition of life is "a self-sustaining chemical system capable of Darwinian evolution," which was adopted by the Exobiology program of NASA (Joyce 1994). In the quest to build a simple living system, much recent interest has focused on encapsulating a genetic or metabolic system inside membrane vesicles (Deamer and Dworkin 2005; Luisi et al. 1999; Morowitz et al. 1988; Ourisson and Nakatani 1994; Szostak et al. 2001). Vesicles are supramolecular aggregates containing an aqueous interior that is separated from the bulk solution by one or more bilayers of amphiphiles.

Why use vesicles? Although they are not strictly required in this definition of life, there are two major reasons why vesicle membranes are thought to be important. The first is that the membrane forms a semipermeable barrier that permits small molecules to pass into the cellular space and traps modified (e.g., phosphorylated or polymerized) products. The second reason is evolutionary: The membrane separates different genomes from one another and reduces the problem of inactive parasites (Szathmary and Demeter 1987; Szostak et al. 2001). During the origin of life, physical grouping is a plausible way for replicator enzymes (replicases) to interact nonrandomly. For example, replicases encapsulated in growing and dividing membrane vesicles would tend to be trapped with sequences related to their own sequence, and thus would preferentially copy those sequences. Because the vesicles separate different genomes from each other, poor replicases would not have access to active replicases, whereas mutants with improved replicase activity would benefit directly themselves, as their descendants remain in the same vesicle and copy each other. An occasional parasitic sequence would

Editors: David Deamer and Jack W. Szostak

Additional Perspectives on The Origins of Life available at www.cshperspectives.org

Copyright (C) 2010 Cold Spring Harbor Laboratory Press; all rights reserved; doi: 10.1101/cshperspect.a002170

Cite this article as Cold Spring Harb Perspect Biol 2010;2:a002170 


\section{I.A. Chen and P. Walde}

be separated from most of the active polymerases during vesicle division and could not poison the entire system (the "stochastic corrector" model) (Smith and Szathmary 1995; Szathmary and Demeter 1987). Thus, a higher level of population organization, the cell, greatly facilitates the evolution of more efficient replicases (Cavalier-Smith 2001; Koch 1984; Matsuura et al. 2002; Szathmary and Demeter 1987; Szostak et al. 2001).

Membrane vesicles are not the only way to segregate different genomes. The attachment of molecules onto surfaces also creates a heterogeneous distribution of interactions based on spatial proximity, a scenario that has been investigated theoretically using cellular automata models (Szabo et al. 2002). Although they may not have been the initial means of achieving genomic segregation during the origin of life, membranes are the dominant means of separating cells today. Membranes presumably assumed this function very long ago, at least three to four billion years ago, at some time before the diversification from the last common ancestor.

Vesicle morphologies and topologies can cover a rich and diverse landscape (Fig. 1, top), although experimentalists tend to prefer unilamellar vesicles because data can be more easily interpreted in this context, and because these vesicles resemble contemporary cells, which

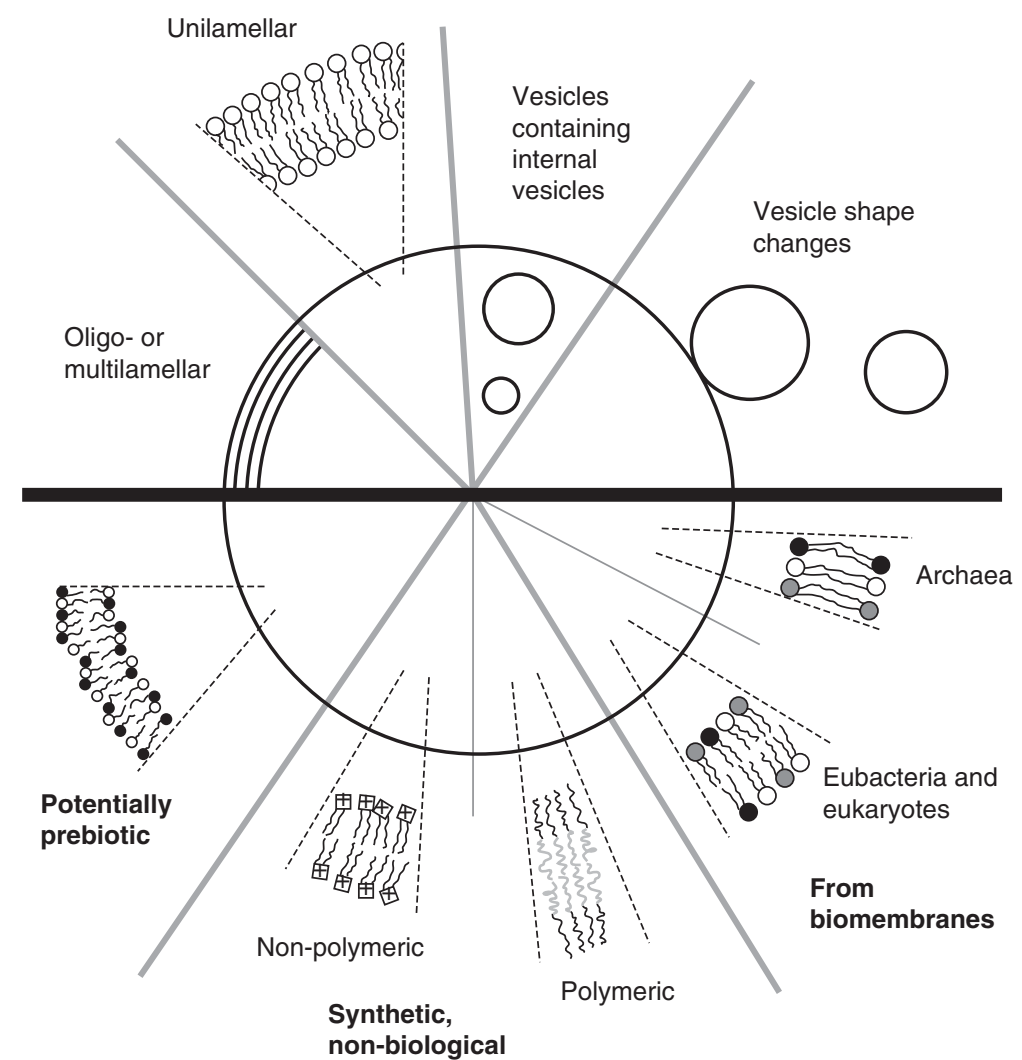

Figure 1. Diversity of morphology and composition of self-assembled vesicles. Top: Schematic representation of possible morphologies and shape changes. Vesicles may be multi-, oligo-, or unilamellar. They may also be multivesicular (containing smaller vesicles inside a large vesicle). Under certain conditions and for certain amphiphiles, vesicle shape changes can be induced (e.g., leading to vesicle budding and fission). Vesicles may also be nonspherical (e.g., tubular). The diameter of vesicles may vary between about $30 \mathrm{~nm}$ and more than $100 \mu \mathrm{m}$. Bottom: Vesicle formation occurs for a large number of chemically diverse amphiphiles, including those naturally occurring in biomembranes as well as completely synthetic amphiphiles. Of particular interest are single-chain amphiphiles (or mixtures of amphiphiles) that are potentially prebiotic. 
use the plasma membrane to separate the cell's interior from the outside environment. The plasma membrane is composed of roughly equal parts protein and lipid amphiphiles, so one might assume that a protocell membrane was also composed of amphiphilic lipids and/ or peptides. However, for simplicity, most experimental work thus far has focused on vesicles made of only one or two prebiotically plausible components (e.g., fatty acid). Vesicles can be made using many different types of amphiphiles, either naturally occurring or synthetic (Fig. 1, bottom). Because of the general robustness of the formation of vesicles, this process has been called an "archetype of self-assembly" (Antonietti and Foerster 2003).

In this article, we first review some fundamentals of self-assembly and focus on important features of vesicles made from single chain amphiphiles. For further discussion of vesicles as model prebiotic experimental systems, we refer the reader to reviews already in the literature (Mansy 2009; Monnard and Deamer 2003; Walde and Ichikawa 2001; Walde et al. 2006) and references contained within these reviews. We then turn to recent results and current challenges in research related to vesicles in the origins of life.

\section{BACKGROUND}

\section{Thermodynamics of Self-Assembly of} Amphiphiles

Self-assembled structures of amphiphiles are the result of a balance of attractive and repulsive forces. Amphiphiles tend to aggregate because of the hydrophobic effect, which stems primarily from the strong attraction of water for itself (Tanford 1973). Nonpolar solutes disrupt the isotropic hydrogen bonding of water, causing an entropic loss at the solute-water interface. Therefore nonpolar molecules tend to aggregate to minimize the interface (direct attractive interactions, such as van der Waals forces, play a relatively small role). In the absence of a repulsive force, the hydrophobic effect would lead to bulkphase separation. However, repulsion among amphiphiles resulting from sterics (e.g., among hydrated head groups) or electrostatics favors the formation of structured assemblages.

A minimum number of molecules is required for effective reduction of the waterhydrophobic surface interface in an aggregate relative to free solution. This leads to a highly cooperative transition in which the concentration of aggregates increases dramatically near a critical aggregation concentration (cac) and for micelles, the critical micellization concentration $(\mathrm{cmc})$. This process is often modeled as a phase transition; although such models are not strictly correct, they often capture the essential behavior of the system within experimental error. This approximation is quite good when aggregates are large (i.e., containing $>50$ molecules), as is the case for most micelles and vesicles.

Micelles are self-assembled structures with a liquidlike hydrocarbon interior and polar headgroups on the exterior (Fig. 2A). Several geometries are possible, including spherical, cylindrical, and ellipsoidal. The free energy of transferring an amphiphile from water into a micelle depends linearly on chain length, so the cmc depends exponentially on chain length (Tanford 1973). Vesicles are closed bilayer structures with an aqueous compartment (Fig. 2B). Whether micelles or vesicles are formed can be explained largely by packing considerations (Israelachvili et al. 1976; Israelachvili et al. 1977). The balance of attractive interactions and repulsive interactions gives an optimal interfacial area per molecule. Amphiphiles selfassemble into structures having area exposed to solvent (a) close to this optimum. Spherical micelles have the greatest interfacial area, in which $v / a=l / 3$ (where $v=$ volume and $l=$ hydrophobic chain length), cylindrical micelles have $v / a=l / 2$, and vesicles have $v / a=l$. To a first approximation, the presence of two chains on an amphiphile doubles $v$ whereas the optimal $a$ and $l$ are constant, so a consequence of these considerations is that single-chain amphiphiles tend to form micelles whereas double-chain amphiphiles tend to form cylindrical micelles or vesicles. Vesicle formation is also favored by factors that reduce the optimal interfacial area. For example, fatty acids ( prebiotically plausible 
I.A. Chen and P. Walde

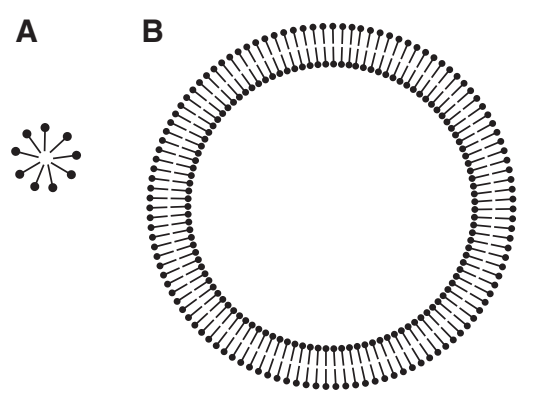

C

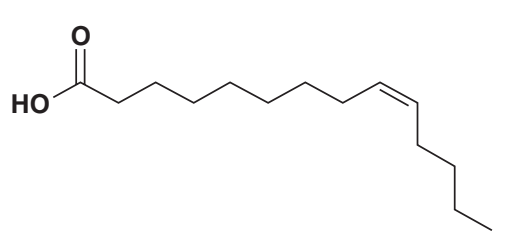

D<smiles>O=C(O)CN(CCO)CCO</smiles>

E

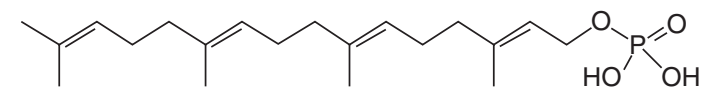

$\mathbf{F}$

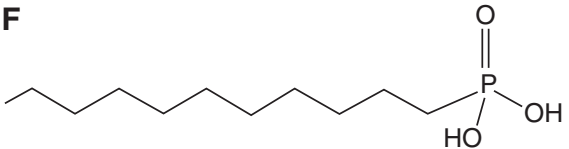

Figure 2. Structures of prebiotically plausible single chain amphiphiles and a commonly used buffer. $(A)$ micelle; $(B)$ vesicle; $(C)$ myristoleic acid; $(D)$ bicine; $(E)$ geranylgeranyl phosphoric acid; $(F) n$-decylphosphonic acid.

single-chain amphiphiles) form micelles at high $\mathrm{pH}$ when their head groups are negatively charged, but they form vesicles at lower $\mathrm{pH}$ when their head groups interact more favorably (Gebicki and Hicks 1973).

Packing constraints also imply a minimum chain length required to form vesicles; for fatty acids (a single chain amphiphile), this length is around eight carbons (Monnard and Deamer 2003). Organic extracts of the Murchison meteorite (Lawless and Yuen 1979) show that abundances of fatty acids decreased as chain length increases, with a three-carbon increase corresponding to a $\sim 10$-fold drop in abundance. Roughly speaking, this drop in abundance is somewhat compensated by the decrease of cac as chain length increases (a three-carbon increase corresponds to a $\sim 10$-fold decrease in cac) (Chen et al. 2006). Although the details of these relationships depend on the synthetic pathway and buffer conditions, this general pattern suggests that, during prebiotic synthesis, each fatty acid would reach its cac at around the same time, leading to a relatively sharp transition to a vesicle world.

\section{Kinetics of Self-Assembly of Amphiphiles}

The kinetics of nucleation and growth of micelles are generally quite fast. Nucleation occurs on the order of microseconds to milliseconds, whereas exchange of monomers through the aqueous phase occurs on the order of nanoseconds to microseconds. These timescales depend on the association and dissociation rates of amphiphiles. As chain length increases, dissociation rates decrease (for alkyl sulphates, roughly 10-fold per three-carbon increase), whereas association rates decrease only slightly because of slower diffusion (within one order of magnitude from C6 to C14) (Aniansson et al. 1976; Hunter 2001).

Vesicle formation from a solution of micelles appears to have two relaxation times, a rapid mixing or aggregation of micelles, followed by slower growth and closure to form vesicles. For a mixture of anionic and zwitterionic surfactant micelles, the micelles mix within a few milliseconds and then coalesce into disclike micelles that grow and close into vesicles within a second (Weiss et al. 2005). For vesicles formed by increasing the $\mathrm{pH}$ of a solution of fatty-acid micelles, aggregation of micelles occurs very quickly $(<12 \mathrm{~ms})$ whereas relaxation into vesicles takes seconds to minutes (Bloechliger et al. 1998; Chen and Szostak 2004a). The kinetics of fatty-acid vesicle formation are particularly interesting with regard to the origins of life for two reasons: (1) the reaction can be autocatalytic, i.e., the presence of vesicles accelerates the formation of more vesicles (Walde et al. 1994b), and (2) vesicle 
formation is catalyzed by many types of surfaces (Hanczyc et al. 2003; Hanczyc et al. 2007a), including montmorillonite, which also catalyzes RNA polymerization from activated monomers (Ferris and Ertem 1992). Once formed, closed vesicles can be fairly stable as supramolecular structures, surviving for several days or longer, even if their components exchange relatively rapidly.

\section{Amphiphiles for Protocells}

The primary components of modern cells are double-chain amphiphiles, particularly phospholipids, which consist of a headgroup and two acyl chains. Because of their geometry, double-chain amphiphiles form stable vesicles very readily. However, they pose several fundamental problems for a protocell, which result from slow dynamics. Phospholipids are relatively impermeable to charged compounds (e.g., $\mathrm{K}^{+}$permeability coefficient: $10^{-10}$ to $10^{-12} \mathrm{~cm} / \mathrm{s}$ (Paula et al. 1996)), possibly because the low mobility of amphiphiles between the leaflets prevents facilitation of transport. Slow flip-flop would also limit vesicle growth by incorporation of new lipid (which requires redistribution of lipid from the outer to inner leaflet), inhibiting a key feature of a self-reproducing system. Modern cells treat their membranes as relatively static barriers, using flipases and permeases to mediate flip-flop and transport, respectively.

On the other hand, single-chain amphiphiles appear to be excellent candidates for prebiotic protocells for several reasons. They have relatively high permeability to ions and small molecules (e.g., $\mathrm{K}^{+}$permeability coefficient: $10^{-6} \mathrm{~cm} / \mathrm{s}$ (Chen and Szostak 2004b)), presumably due at least in part to increased mobility of amphiphiles flipping between the leaflets, facilitating ion passage and circumventing the need for specific transporters. Fast flipflop also permits vesicle growth through addition of an amphiphile feedstock to the vesicle exterior (Berclaz et al. 2001; Chen and Szostak 2004a). Single-chain amphiphiles also have high dissociation rates and relatively high water solubility, so the membrane components can be exchanged among vesicles rapidly, allowing redistribution to "growing" protocells (Chen et al. 2004). The cac is higher for single-chain lipids compared with double-chain lipids of the same chain length and similar head group structure, yielding a greater reservoir of monomers in solution. A limitation of single-chain amphiphiles is that they often form micelles, depending on the molecular geometries and buffer conditions, so the amphiphiles considered for prebiotic vesicles are constrained to have relatively small headgroups compared with double-chain amphiphiles.

Although we do not know which type of amphiphiles constituted the membranes of protocells, much attention has focused on fatty acids (Fig. 2c) and mixtures of fatty acids with their derivatives (e.g., fatty alcohols, amines, and monoacylglycerols). Fatty acids can be synthesized abiotically in several ways. For example, Miller-Urey-type electrical discharge reactions in a solution of ammonia under a nitrogen and methane atmosphere yield fatty acids with a chain length up to C12 (Allen and Ponnamperuma 1967; Yuen et al. 1981). Abiotic syntheses generally yield decreasing amounts of fatty acids of longer chain lengths. A synthesis simulating hydrothermal vents yielded fatty acids up to C33, from an aqueous Fischer-Tropsch-type synthesis using a heated solution of oxalic acid (which disproportionates into $\mathrm{H}_{2}, \mathrm{CO}_{2}$, and CO) (McCollom et al. 1999; Rushdi and Simoneit 2001).

Direct evidence for the abiotic presence of fatty acids comes from the detection of fatty acids in the interior of the Murray and Murchison carbonaceous chondrite meteorites from Australia (up to C8), as well as an Asuka carbonaceous chondrite meteorite (A-881458) from Antarctica (up to C12) (Lawless and Yuen 1979; Naraoka et al. 1999; Yuen et al. 1984; Yuen and Kvenvolden 1973). Fatty acids are relatively abundant in these meteorites, being 20 times more abundant than amino acids in the organic extract of A-881458. Indeed, organic extracts from the Murchison meteorite form boundarymembraneswhen rehydrated(Deamer 1985; Deamer and Pashley 1989). The presence of fatty acids is particularly suggestive because 
the chemical composition of these meteorites is believed to resemble that of the early solar system.

Depending on the solution $\mathrm{pH}$, fatty acids self-assemble into different structures (Cistola et al. 1988; Small 1986). At low pH, the molecules are protonated and uncharged, resulting in an oil phase. At high $\mathrm{pH}$, the molecules are deprotonated and negatively charged, resulting in the formation of micelles with a hydrophobic core and surface-exposed carboxylates that repel one another. Although the $\mathrm{pK}_{\mathrm{a}}$ of a carboxylic acid is typically $4-5$, the self-assembly of fatty acids leads to a cooperative effect that increases the $\mathrm{pK}_{\mathrm{a}}$. For example, oleic acid (C18:1) monomers have a $\mathrm{pK}_{\mathrm{a}}$ of 4.5 , but oleic acid assembled into a bilayer membrane in bicine buffer (Fig. 2D) has a $\mathrm{pK}_{\mathrm{a}}$ of 8.5. Medium- and longchain fatty acids incorporated into membranes have $\mathrm{pK}_{\mathrm{a}} \mathrm{s}$ in the general range of 7-9 (Cistola et al. 1988). When the solution $\mathrm{pH}$ is near the $\mathrm{pK}_{\mathrm{a}}$, fatty acids assemble into bilayer membrane vesicles that are capable of entrapping solutes (Apel et al. 2001; Walde et al. 1994a). These vesicles have a net negative charge, with a formal surface charge density close to half of the molecular density of the membrane. As a result, cations are also associated with the surface, forming an electrical double layer (Grahame 1947; Hunter 2001). Several articles describe in detail the characteristics and limitations of fatty-acid systems (Deamer and Dworkin 2005; Mansy 2009; Monnard et al. 2002; Morigaki and Walde 2007; Walde et al. 2006).

Another category of single-chain amphiphiles that may be promising as a model for prebiotic vesicles uses a phosphate headgroup, such as the polyprenyl phosphates (e.g., geranylgeranyl phosphate; Fig. 2E). These amphiphiles can self-assemble into vesicles (Ourisson and Nakatani 1994; Pozzi et al. 1996). In an interesting parallel to fatty-acid membranes, which can be stabilized to $\mathrm{pH}$ changes by acyl alcohols (Monnard et al. 2002), polyprenyl phosphate membranes are stabilized by addition of polyprenyl alcohols (Streiff et al. 2007). Other phosphate-based systems under investigation include mono- $n$-alkyl phosphates and phosphonates (Walde et al. 1997); alkyl phosphonates in particular have been observed in organic extracts from the Murchison meteorite (e.g., decylphosphonate; Fig. 2F) (Cooper et al. 1992).

\section{RECENT RESULTS}

\section{Membranes and Nucleic Acids}

Vesicles could play a simple role as compartment boundaries for ribozymes (Chen et al. 2005), but they may contribute to an early cell in other ways as well. Because of the importance of the RNA world during origins of life, several groups are studying direct or indirect interactions between vesicle membranes and RNA. One mode of direct interaction between RNA and cationic vesicles is electrostatic attraction to form nucleic acid-lipid complexes (Thomas and Luisi 2005). Such complexes may also be formed with neutral or zwitterionic vesicles under the right buffer conditions (e.g., high divalent cation concentration). For anionic amphiphiles, the electrostatic repulsion between RNA (poly-U) and the vesicles can be overcome by covalently decorating the phopholipid headgroup with adenosine (Milani et al. 2007), analogous to the annealing of two complementary, negatively charged nucleic acid strands. Conversely, the nucleic acid can be conjugated to a hydrophobic moiety, such as cholesterol, which inserts into the membrane and brings the nucleic acid into the proximity of the vesicle (Banchelli et al. 2008). RNA aptamers for membranes have also been found by in vitro selection for RNAs that bound to phospholipid vesicles. These sequences appear to bind the membrane as aggregates (Janas and Yarus 2003). One such sequence was modified to include an RNA motif for tryptophan binding, and together these membrane-binding RNAs had a small but measurable effect on the rate of tryptophan permeation through the membrane (Janas et al. 2004).

Membrane vesicles can also have surprising consequences for the evolution of the RNA world. Membranes can accelerate the polymerization of RNA mononucleotides or amino acids during drying and wetting cycles (Deamer 
et al. 2006; Rajamani et al. 2008; Zepik et al. 2007). This effect may be driven by an increase in local concentration of monomers, through trapping of monomers between lamellae during drying and mass action from dehydration. Although the mechanism is unknown, the liquid crystalline structure of the dried lamellae is believed to be important for this effect.

On the theoretical side, fresh efforts have been made in the analysis of polymerization dynamics relevant to nucleic acids ("prelife"). This mathematical analysis describes the distribution of sequences with and without replication and the occurrence of a phase transition among these regimes (Manapat et al. 2009; Nowak and Ohtsuki 2008). One interesting direction for this research would be consideration in the context of a protocell, to determine how small numbers and competition among protocells might affect the prelife dynamics.

\section{Systems-Level Properties of Protocells}

How did interactions arise between the membrane and its encapsulated contents? Two interactions have been described based solely on the physicochemical properties of the system. The growth of vesicles consists of the incorporation of fatty-acid molecules into the outer leaflet of the membrane, followed by the flip-flop of about half of these molecules into the inner leaflet. Fatty acid flip-flop into the inner leaflet will also transport protons (Hamilton 1998; Kamp and Hamilton 1992), so growth transduces the energy from the micelle-vesicle transition into a $\mathrm{pH}$ gradient across the membrane (Chen and Szostak 2004b). However, proton gradients across fatty-acid membranes only last as long as cations are not transported in the opposite direction, so a cell would need to use the energy stored in the proton gradient quickly before it decayed (Kamp and Hamilton 1992; Zeng et al. 1998). Regardless, the capture of energy is one way the growth of one component, the membrane, could confer an advantage to another component (encapsulated contents).

The emergence of the cell can also be approached from the perspective of the encapsulated genome. A transmembrane gradient of any impermeable solute $(\Delta c)$ exerts osmotic pressure on a semipermeable membrane $(\Delta \Pi=$ $n \Delta c \mathrm{RT}$ for small solutes in dilute solution, where $n=$ number of particles into which the solute dissociates). A charged solute generates more osmotic pressure, and the osmotic pressure of a charged polymer, such as RNA, will be due primarily to the counterions associated with it, in accordance with the Gibbs-Donnan equilibrium (Bartlett and Kromhout 1952; Tinoco et al. 2002). The replication of a genomic polymer would increase the osmotic pressure in a vesicle (assuming that the monomers equilibrate across the membrane). The resulting areal strain favors membrane growth, which relieves the strain by increasing the total area of the membrane. For amphiphiles with high rates of monomer exchange and flip-flop, like fatty acids, this leads to a competition among vesicles for amphiphiles, translating the fitness of the encapsulated replicase into the fitness of the whole cell (Chen et al. 2004). Darwinian competition between "cells" could have emerged simply on encapsulation of replicases or metabolic cycles inside vesicles; empty vesicles and vesicles encapsulating less efficient systems would shrink by losing membrane.

This competition has interesting implications for the natural selection of chemical traits in the genome and membrane. Because counterions cause most of the change in osmotic pressure after polymerization, protocells containing charged polymers as the genetic material, such as RNA or DNA, would outcompete those with neutral polymers (e.g., peptide nucleic acid). With respect to the membrane, the protocellular competition might favor the evolution of an enzyme that stabilized the membrane against loss of amphiphiles, such as by condensing two fatty-acid molecules together. In turn, the reduced permeability and growth dynamics of stabilized membranes might drive the coevolution of transporters and more enzymes. Thus the competition arising from the simple physical properties of an encapsulated replicase could result in a "snowball" effect on the evolution of cellular complexity. Such predictions may suggest further experimental exploration. 


\section{Computer Simulation of \\ Protocell-Like Systems}

In an era of increasingly powerful and accessible computation, molecular dynamics (MD) simulation is a promising technique for exploring our understanding of the behavior of relatively large chemical systems like vesicles. In general these simulations use a combination of empirical and $a b$ initio information to describe energy potentials for molecular conformations and intermolecular forces (dispersion and electrostatic forces) and solve the Newtonian equations of motion numerically. The size of a vesicle system is roughly hundreds of thousands to a few millions of atoms (e.g., a single fatty acid is roughly 50 atoms, and thousands of fatty-acid molecules would compose a small vesicle, and water would also compose a substantial mole fraction of the system). Currently an MD simulation of this size could simulate about 100 nanoseconds, which would be enough to study the fast kinetics of self-assembly. An atomistic simulation of dipalmitoyl phosphatidylcholine (DPPC) in water recapitulated vesicle selfassembly (de Vries et al. 2004). In addition to testing our understanding of the system, ideally these simulations could be useful to experimentalists for building biophysical intuition and emphasizing features to be further explored. For example, the simulation of a DPPC vesicle indicated a large asymmetry in mass and ordering between the inner and outer bilayers, suggesting a potential mechanism for asymmetric insertion of molecules into the membrane.

Slower dynamics or larger systems could be modeled using coarse-grained rather than atomistic simulations (Klein and Shinoda 2008). One interesting but poorly understood phenomenon that might benefit from MD simulation is the recent observation that dried lipid membranes accelerate the polymerization of amino acids and nucleotide monophosphates, perhaps by preorganizing the reactants into the reactive geometry (Rajamani et al. 2008).

Another style of simulation of protocells focuses on the reaction (or reaction-diffusion) kinetics of the system without modeling the molecular details. Although much work, such as Manfred Eigen's classic model of hypercycles (Eigen 1971), dealt with metabolic cycles without explicit reference to a membrane, recently the potential for interactions between the membrane and encapsulated metabolic reactions has come under closer scrutiny (Mavelli and RuizMirazo 2007). Stochastic simulations are of particular interest because the volume of a small vesicle could be less than one attoliter, such that micromolar concentrations would give only a handful of molecules per vesicle. Although care is always required when dealing with assumptions in a model, simulations can provide a testing ground for our understanding, and may become a useful tool for understanding experimental results and generating interesting, experimentally testable predictions in protocellsized systems.

\section{CHALLENGES AND FUTURE DIRECTIONS}

\section{Physical Forces and Protocells}

How would protocells interact with their environment? Although some interest has focused on interactions at a molecular level, such as the permeability and thermostability of vesicles (Mansy et al. 2008; Mansy and Szostak 2008), less work has been performed to relate colloidal processes to protocells. For example, transport phenomena might give us clues about protocell movement through a heterogeneous environment or even initially homogeneous environment. Hanczyc et al. showed how oleic anhydride oil droplets added to an alkaline solution spontaneously move, as exothermic hydrolysis of the anhydride sets up a convective current within the droplet; the direction of motion appeared to be the outcome of symmetry-breaking fluctuation (Hanczyc et al. 2007b). The theory of osmophoresis suggests that vesicles in a solute gradient will move in the direction of lower solute concentration (Anderson 1983; Nardi et al. 1999). Such effects, if experimentally relevant, could inform our understanding of how a protocell would move within its environment as a consequence of colloidal forces, without motor machinery. 
Entropy might play a very interesting role in protocells. For example, theory predicts that two chromosomes in a cell tend to segregate from each other to maximize conformational entropy (Jun and Mulder 2006). This finding suggests that protocell genomes might not require extra machinery to segregate its genetic material. Along similar lines, cell division machinery (in particular, a contractile ring) was recently discovered to be unnecessary for reproduction and division of Bacillus subtilis (Leaver et al. 2009). The observed mode of division is reminiscent of a physical phenomenon, the Rayleigh instability (also known as "pearling" in tubular liposomes), lending credence to the idea that protocells would not require division machinery (Chen 2009). One might also expect protocells to interact with one another through entropic effects, such as the depletion effect, in which the entropy of the aqueous solution is maximized when large particles are close together, resulting in an effective attraction between the particles. Such effects could be interesting as leading to assembly of protocells into larger structures. Investigation of the role of physical forces in protocells may present many opportunities for collaboration between traditional soft condensed matter physicists and origins of life researchers.

\section{Vesicles from Mixtures}

Studies on vesicles formed from potentially prebiotic amphiphiles have so far been limited to systems containing one or two types of amphiphiles. This is in contrast to the output of simulated prebiotic chemical reactions, which typically produce very heterogeneous mixtures of compounds. In addition, little experimental work has been performed to integrate peptides and lipids. (A notable exception to this bias toward working with one or two component systems was a study using the heterogeneous organic extract from a carbonaceous chondrite meteorite to form vesicles [Deamer 1985].) The reason for this bias is mainly the avoidance of analytical difficulties, but clearly it is not very realistic.

What properties might vary with membrane composition? Several features characterize a particular vesicle system and differentiate it from others, including (1) lamellarity, (2) average membrane thickness and its fluctuations, (3) amphiphiledynamicsin themembrane, (4) membrane permeability, i.e., the ability to keep solutes captured inside the vesicles and to stabilize concentration gradients across the membrane, (5) chemical stability of the amphiphiles, (6) membrane surface charge, (7) membrane fluidity, (8) possibility of membrane budding and fission (leading to vesicle divisions), (9) vesicle aggregation or vesicle adsorption to surfaces, (10) vesicle fusion, (11) colloidal stability at different temperatures and bulk solution compositions. All these properties ultimately depend on the chemical structure of the amphiphiles, the environmental conditions, and the process of vesicle formation. The process of vesicle formation is relevant because most vesicles are kinetically trapped systems (i.e., not at true thermodynamic equilibrium). Lamellarity and size depend strongly on how the vesicles are prepared, but the other features depend primarily on the vesicle composition and buffer conditions.

There are challenges to using vesicles based on fatty acids as protocell models (Monnard and Deamer 2002). One limitation for practical studies is the failure in preparing vesicles from long-chain fatty acids at room temperature, for example from hexadecanoic acid, because of the high melting temperature of saturated linear hydrocarbon chains. According to the phase diagram, a lamellar phase exists at $70^{\circ} \mathrm{C}$ (Skurtveit et al. 1989). However, recently it was shown that vesicles form from long-chain fatty acids at room temperature if guanidine hydrochloride is added (Douliez et al. submitted for publication). This illustrates how vesicles of mixed composition can actually have unanticipated properties beneficial to a protocell, such as robustness to ionic strength, lowered cac, or even propagation of information (as discussed later) (Monnard and Deamer 2003). One of the challenges for the future will be to further study these mixtures to understand the chemical basis for their effects on vesicle characteristics. Other mixtures to explore include mixtures of fatty acids with small molecule components, mixtures of different types 
of fatty acids, and mixtures of fatty acids with peptides.

\section{Self-Assembled Structures and Information}

The storage and propagation of information is a key feature of biological systems. Nucleic acid sequences fulfill this requirement in a straightforward fashion, particularly in the case of ribozymes, where the informational material itself has chemical activity. However, in a very broad sense, molecules other than nucleic acids carry information about their reactivity and physicochemical properties (Graham et al. 2004). Selfassembling molecules contain the information for building supramolecular structures, such as vesicles and micelles. For example, potassium decanoate, $\mathrm{CH}_{3}\left(\mathrm{CH}_{2}\right)_{6} \mathrm{COO}^{-} \mathrm{K}^{+}$, carries information on the thermodynamics of its selfassembly behavior. When dissolved in water at a concentration of $29.1 \mathrm{~g} / \mathrm{L}$ (150 mM), micellar aggregates form (Namani and Walde 2005). Each micelle contains a hydrophobic core that allows some solubilization of molecules that are not soluble in water, so the self-assembly of the amphiphilic decanoate molecules confers new physicochemical properties to the aqueous solution (Luisi 2002). Could self-assembled, noncovalent systems also propagate their "information"?

An interesting model addressing this question was proposed recently (Segre et al. 2000). In a solution containing a mixture of amphiphiles, if amphiphiles of a certain type favored the incorporation of other amphiphiles in a selective fashion, an assemblage would reach a particular steady state whose composition contains some information. In simulations they observed that abrupt transitions could occur among different steady states, suggesting that different compositions could coexist and thereby provide variation on which natural selection might act. An experimental system corresponding to this model would require determining the on- and off- rates of different amphiphiles in vesicles spanning a range of compositions. A particularly interesting technical challenge associated with this theoretical problem is the identification of the composition of an individual vesicle.
Self-assembly as a Quantitative Trait

Although one working definition of life (a selfsustaining chemical reaction capable of Darwinian evolution) was given in the introduction to this article, alternative definitions also exist, often in the form of several criteria. The difficulties encountered in defining life suggest that life is a quantitative, not qualitative, trait, which could be measured by an appropriate metric. Chirikjian and coworkers developed metrics for the degree of self-replication and task complexity in a framework of self-replicating robots (Lee et al. 2008). For example, some robots copy themselves by joining two already complex building blocks (low self-replication) in a highly structured environment with many landmarks (low task complexity), whereas other robots copy themselves by joining several simple building blocks (high self-replication) in an unstructured environment (high task complexity). These calculations require knowledge of the components, their interconnections, and the environment. Application of this quantitative approach to chemical systems and to other properties (i.e., evolvability and life) poses an interesting intellectual challenge that might also help integrate our understanding of different model systems of protocells.

\section{REFERENCES}

Allen WV, Ponnamperuma C. 1967. A possible prebiotic synthesis of monocarboxylic acids. Currents Mod Biol 1: 24-28.

Anderson JL. 1983. Movement of a semipermeable vesicle through an osmotic gradient. Phys Fluids 26: 28712879.

Aniansson EAG, Wall SN, Almgren M, Hoffmann $\mathrm{H}$, Kielmann I, Ulbricht W, Zana R, et al. 1976. Theory of kinetics of micellar equilibria and quantitative interpretation of chemical relaxation studies of micellar solutions of ionic surfactants. J Phys Chem 80: 905-922.

Antonietti M, Foerster S. 2003. Vesicles and liposomes: A self-assembly principle beyond lipids. Adv Materials 15: $1323-1333$.

Apel CL, Deamer DW, Mautner MN. 2001. Self-assembled vesicles of monocarboxylic acids and alcohols: Conditions for stability and for the encapsulation of biopolymers. Biochim Biophys Acta 1559: 1-9.

Banchelli M, Betti F, Berti D, Caminati G, Bombelli FB, Brown T, Wilhelmsson LM, et al. 2008. Phospholipid membranes decorated by cholesterol-based oligonucleotides 
as soft hybrid nanostructures. J Phys Chem B 112: $10942-$ 10952.

Bartlett JH, Kromhout RA. 1952. The Donnan equilibrium. Bull Math Biophys 14: 385-391.

Berclaz N, Muller M, Walde P, Luisi PL. 2001. Growth and transformation of vesicles studied by ferritin labeling and cryotransmission electron microscopy. J Phys Chem B 105: 1056-1064.

Bloechliger E, Blocher M, Walde P, Luisi PL. 1998. Matrix effect in the size distribution of fatty acid vesicles. J Phys Chem B 102: 10383-10390.

Cavalier-Smith T. 2001. Obcells as proto-organisms: Membrane heredity, lithophosphorylation, and the origins of the genetic code, the first cells, and photosynthesis. J Mol Evol 53: 555-595.

Chen IA. 2009. Cell division: Breaking up is easy to do. Curr Biol 19: R327-R328.

Chen IA, Szostak JW. 2004a. A kinetic study of the growth of fatty acid vesicles. Biophys. J. 87: 988-998.

Chen IA, Szostak JW. 2004b. Membrane growth can generate a transmembrane $\mathrm{pH}$ gradient in fatty acid vesicles. Proc. Natl. Acad. Sci 101: 7965-7970.

Chen IA, Roberts RW, Szostak JW. 2004. The emergence of competition between model protocells. Science 305: 1474-1476.

Chen IA, Salehi-Ashtiani K, Szostak JW. 2005. RNA catalysis in model protocell vesicles. J. Am. Chem. Soc. 127: $13213-13219$.

Chen IA, Hanczyc MM, Sazani PL, Szostak JW. 2006. Protocells: Genetic polymers inside membrane vesicles. In Atkins J.F. ed. RNA World, 3rd ed., Cold Spring Harbor Laboratory Press.

Cistola DP, Hamilton JA, Jackson D, Small DM. 1988. Ionization and phase behavior of fatty acids in water: Application of the Gibbs phase rule. Biochemistry 27: 1881-1888.

Cooper GW, Onwo WM, Cronin JR. 1992. Alkyl phosphonic-acids and sulfonic-acids in the Murchison meteorite. Geochimica Et Cosmochimica Acta 56: 4109-4115.

de Vries AH, Mark AE, Marrink SJ. 2004. Molecular dynamics simulation of the spontaneous formation of a small DPPC vesicle in water in atomistic detail. J Am Chem Soc 126: $4488-4489$.

Deamer DW. 1985. Boundary structures are formed by organic components of the Murchison carbonaceous chondrite. Nature 317: 792-794.

Deamer DW, Pashley RM. 1989. Amphiphilic components of the Murchison carbonaceous chondrite: Surface properties and membrane formation. Orig Life Evol Biosph 19: 21-38.

Deamer DW, Dworkin JP. 2005. Chemistry and physics of primitive membranes, Pages 1-27. In: Walde P. ed. Prebiotic chemistry: From simple amphiphiles to protocell models. Topics in Current Chemistry.

Deamer D, Singaram S, Rajamani S, Kompanichenko V, Guggenheim S. 2006. Self-assembly processes in the prebiotic environment. Phil Trans Roy Soc B-Biol Sci 361: $1809-1818$.

Eigen M. 1971. Selforganization of matter and the evolution of biological macromolecules. Naturwissenschaften 58: 465-523.
Ferris JP, Ertem G. 1992. Oligomerization of ribonucleotides on montmorillonite: reaction of the $5^{\prime}$-phosphorimidazolide of adenosine. Science 257: 1387-1389.

Gebicki JM, Hicks M. 1973. Ufasomes are stable particles surrounded by unsaturated fatty acid membranes. Nature 243: $232-234$.

Graham DJ, Malarkey C, Schulmerich MV. 2004. Information content in organic molecules: Quantification and statistical structure via Brownian processing. J Chem Inf Computer Sci 44: 1601-1611.

Grahame DC. 1947. The electrical double layer and the theory of electrocapillarity. Chem Rev 41: 441-501.

Hamilton JA. 1998. Fatty acid transport: Difficult or easy? J Lipid Res 39: 467-481.

Hanczyc MM, Fujikawa SM, Szostak JW. 2003. Experimental models of primitive cellular compartments: encapsulation, growth, and division. Science 302: 618-622.

Hanczyc MM, Mansy SS, Szostak JW. 2007a. Mineral surface directed membrane assembly. Origins of Life and Evolution of the Biosphere 37: 67-82.

Hanczyc MM, Toyota T, Ikegami T, Packard N, Sugawara T. 2007b. Fatty acid chemistry at the oil-water interface: Self-propelled oil droplets. J Am Chem Soc 129: 9386-9391.

Hunter RJ. 2001. Foundations of colloid science. New York, Oxford University Press.

Israelachvili JN, Mitchell DJ, Ninham BW. 1976. Theory of self-assembly of hydrocarbon amphiphiles into micelles and bilayers. J Chem Soc Faraday Trans II 72: 1525-1568.

Israelachvili JN, Mitchell DJ, Ninham BW. 1977. Theory of self-assembly of lipid bilayers and vesicles. Biochim Biophys Acta 470: 185-201.

Janas T, Yarus M. 2003. Visualization of membrane RNAs. Rna-a Publication of the Rna Society 9: 1353-1361.

Janas T, Janas T, Yarus M. 2004. A membrane transporter for tryptophan composed of RNA. Rna-a Publication of the Rna Society 10: 1541-1549.

Joyce G. 1994. Foreword. In: Deamer D.W., Fleischaker G.R. eds. Origins of life: The central concepts. Boston, Jones and Bartlett Publishers.

Jun S, Mulder B. 2006. Entropy-driven spatial organization of highly confined polymers: Lessons for the bacterial chromosome. Proc Natl Acad Sci 103: 12388-12393.

Kamp F, Hamilton JA. 1992. pH gradients across phospholipid membranes caused by fast flip-flop of un-ionized fatty acids. Proc Natl Acad Sci 89: 11367-11370.

Klein ML, Shinoda W. 2008. Large-scale molecular dynamics simulations of self-assembling systems. Science 321: 798-800.

Koch AL. 1984. Evolution vs the number of gene copies per primitive cell. J Mol Evol 20: 71-76.

Lawless JG, Yuen GU. 1979. Quantification of monocarboxylic acids in the Murchison carbonaceous meteorite. Nature 282: 396-398.

Leaver M, Dominguez-Cuevas P, Coxhead JM, Daniel RA, Errington J. 2009. Life without a wall or division machine in Bacillus subtilis. Nature 457: 849-853.

Lee K, Moses M, Chirikjian GS. 2008. Robotic Self-replication in structured environments: Physical demonstrations and complexity measures. Int J Robotics Res 27: 387. 
I.A. Chen and P. Walde

Luisi PL. 2002. Emergence in chemistry: Chemistry as the embodiment of emergence. Foundations Chem 4: 183200.

Luisi PL, Walde P, Oberholzer T. 1999. Lipid vesicles as possible intermediates in the origin of life. Current Opinion Colloid Interface Sci 4: 33-39.

Manapat M, Ohtsuki H, Burger R, Nowak MA. 2009. Originator dynamics. J Theoret Biol 256: 586-595.

Mansy SS. 2009. Model protocells from single-chain lipids. Int J Mol Sci 10: 835-843.

Mansy SS, Szostak JW. 2008. Thermostability of model protocell membranes. Proc Natl Acad Sci 105: 13351-13355.

Mansy SS, Schrum JP, Krishnamurthy M, Tobe S, Treco DA, Szostak JW. 2008. Template-directed synthesis of a genetic polymer in a model protocell. Nature 454:p122-U110.

Matsuura T, Yamaguchi M, Ko-Mitamura EP, Shima Y, Urabe I, Yomo T. 2002. Importance of compartment formation for a self-encoding system. Proc Natl Acad Sci 99: 7514-7517.

Mavelli F, Ruiz-Mirazo K. 2007. Stochastic simulations of minimal self-reproducing cellular systems. Philosophical Trans Roy Soc B-Biological Sciences 362: 1789-1802.

McCollom TM, Ritter G, Simoneit BR. 1999. Lipid synthesis under hydrothermal conditions by Fischer-Tropsch-type reactions. Orig. Life Evol. Biosph. 29: 153-166.

Milani S, Bombelli FB, Berti D, Baglioni P. 2007. Nucleolipoplexes: A new paradigm for phospholipid bilayernucleic acid interactions. J Am Chem Soc 129: 11664-+.

Monnard PA, Deamer DW. 2002. Membrane self-assembly processes: Steps toward the first cellular life. Anat Rec 268: 196-207.

Monnard PA, Deamer DW. 2003. Preparation of vesicles from nonphospholipid amphiphiles. Meth Enzymol 372: $133-151$.

Monnard PA, Apel CL, Kanavarioti A, Deamer DW. 2002. Influence of ionic inorganic solutes on self-assembly and polymerization processes related to early forms of life: Implications for a prebiotic aqueous medium. Astrobiology 2: 139-152.

Morigaki K, Walde P. 2007. Fatty acid vesicles. Current Opinion in Colloid Interface Sci 12: 75-80.

Morowitz HJ, Heinz B, Deamer DW. 1988. The chemical logic of a minimum protocell. Orig Life Evol Biosph 18: 281-287.

Namani T, Walde P. 2005. From decanoate micelles to decanoic acid/dodecylbenzenesulfonate vesicles. Langmuir 21: $6210-6219$.

Naraoka H, Shimoyama A, Harada K. 1999. Molecular distribution of monocarboxylic acids in Asuka carbonaceous chondrites from Antarctica. Orig Life Evol Biosph 29: $187-201$.

Nardi J, Bruinsma R, Sackmann E. 1999. Vesicles as Osmotic Motors. Phys Rev Lett 82:5168-5171.

Nowak MA, Ohtsuki H. 2008. Prevolutionary dynamics and the origin of evolution. Proc Natl Acad Sci 105: 1492414927.

Ourisson G, Nakatani Y. 1994. The terpenoid theory of the origin of cellular life: the evolution of terpenoids to cholesterol. Chem Biol 1: 11-23.
Paula S, Volkov AG, Van Hoek AN, Haines TH, Deamer DW. 1996. Permeation of protons, potassium ions, and small polar molecules through phospholipid bilayers as a function of membrane thickness. Biophys J 70: 339-348.

Pozzi G, Birault V, Werner B, Dannenmuller O, Nakatani Y, Ourisson G, Terakawa S. 1996. Single-chain polyprenyl phosphates form "primitive" membranes. Angewandte Chemie-International Edition 35: 177-180.

Rajamani S, Vlassov A, Benner S, Coombs A, Olasagasti F, Deamer D. 2008. Lipid-assisted synthesis of RNA-like polymers from mononucleotides. Orig Life Evol Biosph 38: $57-74$.

Rushdi AI, Simoneit BR. 2001. Lipid formation by aqueous Fischer-Tropsch-type synthesis over a temperature range of 100 to 400 degrees C. Orig. Life Evol. Biosph. 31: 103118.

Segre D, Ben-Eli D, Lancet D. 2000. Compositional genomes: Prebiotic information transfer in mutually catalytic noncovalent assemblies. Proc Natl Acad Sci 97: 4112-4117.

Skurtveit R, Sjoblom J, Hoiland H. 1989. Emulsions under Elevated-Temperature and Pressure Conditions. 1. The Model System Water Hexadecanoic Acid Sodium Hexadecanoate Decane at 70-Degrees-C. J Colloid Interface Sci 133: 395-403.

Small DM. 1986. Chapter 9, Pages 285-343. In: Small D.M. ed. The physical chemistry of lipids: from alkanes to phospholipids. Handbook of lipid research. New York, Plenum Press.

Smith JM, Szathmary E. 1995. The major transitions in evolution. Oxford, W.H. Freeman.

Streiff S, Ribeiro N, Wu ZY, Gumienna-Kontecka E, Elhabiri M, Albrecht-Gary AM, Ourisson G, et al. 2007. "Primitive" membrane from polyprenyl phosphates and polyprenyl alcohols. Chem Biol 14: 313-319.

Szabo P, Scheuring I, Czaran T, Szathmary E. 2002. In silico simulations reveal that replicators with limited dispersal evolve towards higher efficiency and fidelity. Nature 420: 340-343.

Szathmary E, Demeter L. 1987. Group selection of early replicators and the origin of life. J Theor Biol 128: 463-486.

Szostak JW, Bartel DP, Luisi PL. 2001. Synthesizing life. Nature 409: 387-390.

Tanford C. 1973. The hydrophobic effect: formation of micelles and biological membranes. New York, John Wiley and Sons, Inc.

Thomas CF, Luisi PL. 2005. RNA selectively interacts with vesicles depending on their size. J Phys Chem B 109: 14544-14550.

Tinoco I, Sauer K, Wang JC, Puglisi JD. 2002. Physical Chemistry: Principles and applications in biological sciences. Upper Saddle River, New Jersey, Prentice Hall.

Walde P, Ichikawa S. 2001. Enzymes inside lipid vesicles: Preparation, reactivity and applications. Biomol Eng 18: 143-177.

Walde P, Goto A, Monnard P-A, Wessicken M, Luisi PL. 1994a. Oparin's reactions revisited: enzymatic synthesis of poly(adenylic acid) in micelles and self-reproducing vesicles. J Am Chem Soc 116: 7541-7547.

Walde P, Namani T, Morigaki K, Hauser H. 2006. Formation and Properties of Fatty Acid Vesicles (Liposomes), Pages 
1-19. In: Gregoriadis G. ed. Liposome Technology. New York, Informa Healthcare.

Walde P, Wessicken M, Radler U, Berclaz N, CondeFrieboes K, Luisi PL. 1997. Preparation and characterization of vesicles from mono-n-alkyl phosphates and phosphonates. J Phys Chem B 101: 7390-7397.

Walde P, Wick R, Fresta M, Mangone A, Luisi PL. 1994b. Autopoietic self-reproduction of fatty acid vesicles. J Am Chem Soc 116: 11649-11654.

Weiss TM, Narayanan T, Wolf C, Gradzielski M, Panine P, Finet S, Helsby WI. 2005. Dynamics of the self-assembly of unilamellar vesicles. Phys Rev Lett 94: 038303.

Yuen GU, Kvenvolden KA. 1973. Monocarboxylic acids in Murray and Murchison carbonaceous meterorites. Nature 246: $301-303$.
Yuen GU, Lawless JG, Edelson EH. 1981. Quantification of monocarboxylic acids from a spark discharge synthesis. J Mol Evol 17: 43-47.

Yuen G, Blair N, Des Marais DJ, Chang S. 1984. Carbon isotope composition of low molecular weight hydrocarbons and monocarboxylic acids from Murchison meteorite. Nature 307: 252-254.

Zeng Y, Han X, Schlesinger P, Gross RW. 1998. Nonesterified fatty acids induce transmembrane monovalent cation flux: Host-guest interactions as determinants of fatty acid-induced ion transport. Biochemistry 37: 9497-9508

Zepik HH, Rajamani S, Maurel MC, Deamer D. 2007. Oligomerization of thioglutamic acid: Encapsulated reactions and lipid catalysis. Orig Life Evol Biosph 37: 495505. 


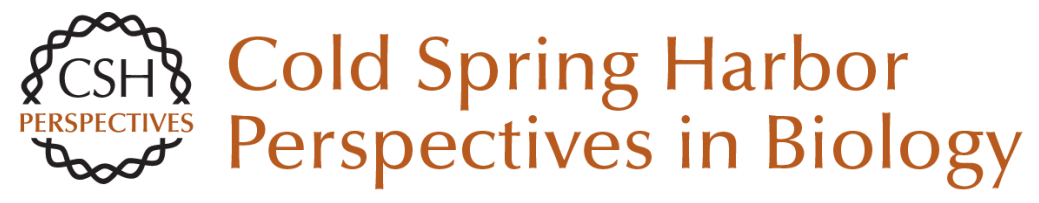

\section{From Self-Assembled Vesicles to Protocells}

Irene A. Chen and Peter Walde

Cold Spring Harb Perspect Biol 2010; doi: 10.1101/cshperspect.a002170 originally published online June 2, 2010

\section{Subject Collection The Origins of Life}

Constructing Partial Models of Cells

Norikazu Ichihashi, Tomoaki Matsuura, Hiroshi Kita, et al.

Ribonucleotides John D. Sutherland

Deep Phylogeny--How a Tree Can Help

Characterize Early Life on Earth

Eric A. Gaucher, James T. Kratzer and Ryan N. Randall

Cosmic Carbon Chemistry: From the Interstellar Medium to the Early Earth

Pascale Ehrenfreund and Jan Cami

Origin and Evolution of the Ribosome George E. Fox

Planetary Organic Chemistry and the Origins of

Biomolecules

Steven A. Benner, Hyo-Joong Kim, Myung-Jung Kim, et al.

Mineral Surfaces, Geochemical Complexities, and the Origins of Life

Robert M. Hazen and Dimitri A. Sverjensky

Historical Development of Origins Research Antonio Lazcano
The Hadean-Archaean Environment

Norman H. Sleep

An Origin of Life on Mars

Christopher P. McKay

Primitive Genetic Polymers

Aaron E. Engelhart and Nicholas V. Hud

Membrane Transport in Primitive Cells Sheref S. Mansy

The Origins of Cellular Life Jason P. Schrum, Ting F. Zhu and Jack W. Szostak

From Self-Assembled Vesicles to Protocells Irene A. Chen and Peter Walde

The Origin of Biological Homochirality Donna G. Blackmond

Earth's Earliest Atmospheres Kevin Zahnle, Laura Schaefer and Bruce Fegley

For additional articles in this collection, see http://cshperspectives.cshlp.org/cgi/collection/

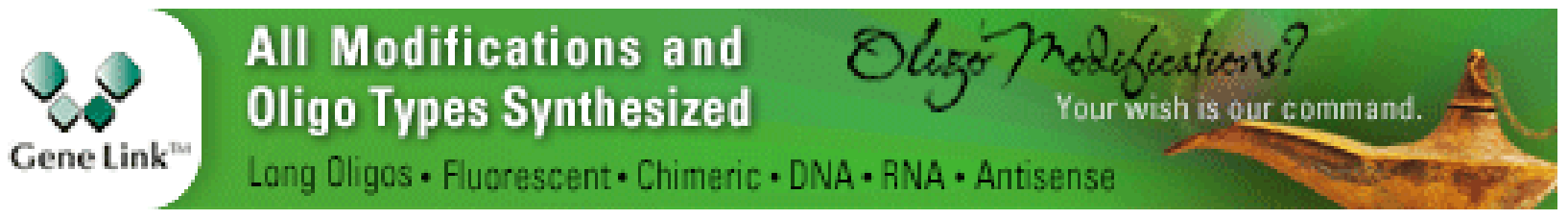

Copyright @ 2010 Cold Spring Harbor Laboratory Press; all rights reserved 